\title{
Artificial Intelligence System for College Students' Physical Fitness and Health Management Based on Physical Measurement Big Data
}

\author{
Li $\mathbf{A i}\left(^{1,2}\right.$ \\ ${ }^{1}$ College of Physical Education, University of Sanya, Sanya 572022, China \\ ${ }^{2}$ College of General Education, Guangxi University of Technology (Preparatory), Guigang 537100, China
}

Correspondence should be addressed to Li Ai; hxciaili@126.com

Received 3 August 2021; Revised 24 August 2021; Accepted 25 August 2021; Published 21 September 2021

Academic Editor: Yuanpeng Zhang

Copyright (c) $2021 \mathrm{Li} \mathrm{Ai}$. This is an open access article distributed under the Creative Commons Attribution License, which permits unrestricted use, distribution, and reproduction in any medium, provided the original work is properly cited.

\begin{abstract}
Most of the current health management products are used in medical institutions and generally do not pay enough attention to the student population. Based on this, this paper designs a student-oriented and functional autonomous health management system. This paper proposes a personal health management system based on a multidimensional data model based on the main social characteristics of the population with chronic diseases and the actual needs of personal health management for chronic diseases. The value of various health data for health management is deeply analyzed and mined, and a multidimensional model data warehouse is constructed according to relevant national health data standards to create a standard data platform for intelligent health warning and disease risk assessment. This paper researches and designs a closed-loop personal health management method based on the Plan-Do-Check-Action (PDCA) cycle management model, with detailed functional design in four aspects: health data collection and recording, health assessment, health planning, and tracking and execution. This paper researches health data collection, processing, and storage technologies and adopts HDFS data storage technology, html, css, Java Script, java, and other software development technologies, combined with j Query, UEditor, Date Range Picker, and other plug-ins, as well as SMS email generation interface, wireless Bluetooth transmission interface, etc. This system web and mobile application platforms are designed and developed. Relational database is used as the system database, and a snowflaketype multidimensional data model is designed. Finally, the functions and performance of this system were tested, and the development and trial run of the basic version have been completed.
\end{abstract}

\section{Introduction}

As population continues to age, people's lifestyles and environments become more complex, leading to a very large population of chronic diseases, increasingly serious subhealth problems, and rising cancer incidence and mortality [1]. The 2018 joint study on medical reform released by the National Health and Family Planning Commission states that nearly 300 million people suffer from chronic diseases. Chronic diseases have become the main cause of death among residents [2]. Statistics show that deaths from chronic diseases account for $79.5 \%$ and $85.3 \%$ of the total deaths in rural and urban areas in China, respectively. The main sources of death from chronic diseases are cardiovas- cular diseases, cancer, and slow respiratory diseases, among which the proportion of hypertension, diabetes, and slow respiratory diseases are $25.2 \%, 9.7 \%$, and $9.9 \%$, respectively, which shows that the harm of chronic diseases is serious and long-term [3]. With the increasing improvement of people's living and cultural standards, people are also paying more and more attention to personal health management, gradually shifting the focus of personal health management from illness and then medical treatment to prevention-based continuous long-term self-health management [4]. According to the World Health Organization statistics, the main factors leading to chronic diseases and their proportion are lifestyle $60 \%$, genetic $15 \%$, social $10 \%$, medical $8 \%$, and climatic $7 \%$. Therefore, a good lifestyle can reduce the prevalence of 


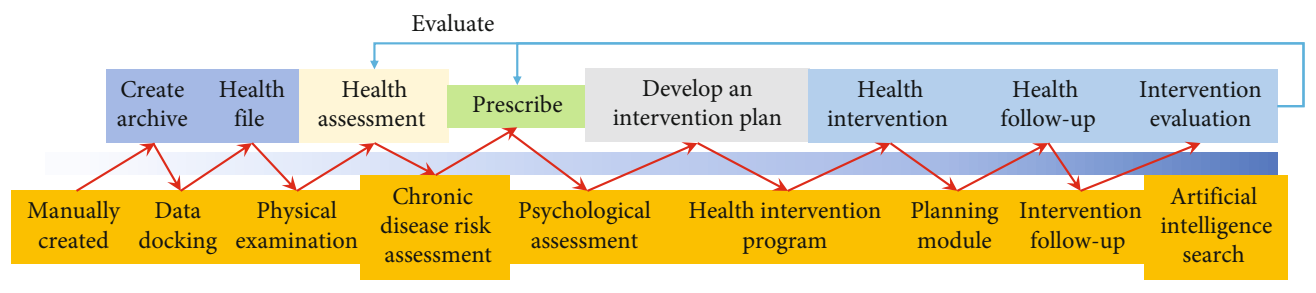

FIgURE 1: Health management process.

chronic diseases or improve the worsening trend of existing chronic diseases.

To develop a good lifestyle, people need comprehensive and accurate information about their health, long-term tracking of their physical condition, and timely intervention to correct poor lifestyles, of which comprehensive and accurate health information is the most important part [5]. The lack of transparency and asymmetry of medical information makes it difficult for individuals to participate in medical and health management, and it is easy to generate excessive medical care and waste medical resources [6]. As mentioned above, if people want to participate in the whole process of their own health management continuously in the long term, the temporary data obtained from medical visits under the traditional model is far enough to meet people's demand for health management. With the rapid advancement of computer technology, the gradual popularization of home medical devices, and the introduction of national health China 2030 and other information policies, the "Internet + health care" health service model has been developed rapidly, and personal health management systems have been created [7]. Personal health management system can be understood as a computer application that can prevent and control the occurrence and development of physical diseases, reduce medical costs, improve the quality of life and self-management awareness, improve lifestyle-related health risk factors, and make the long-term continuous process of personal health management in a safe and confidential environment. Health information collection, health testing, health assessment, health planning, and health intervention are the main means to continuously improve health status. The purpose is to prevent the occurrence and development of chronic noncommunicable diseases, improve the quality of life, and reduce medical costs [8]. The personal health management system is a perfect combination of advanced information technology and medical technology, mainly highlighting the individual as the center; the system assists individual users to carry out self-health management, so that health management can be carried out scientifically and on the basis [9]. It mainly helps healthy people and subhealthy people to establish an orderly and healthy lifestyle by maintaining health and promoting health (as shown in Figure 1). By collecting and recording personal health information, the system then cleans, stores, and analyzes user's health data, which can ensure the accuracy and integrity of the health data throughout the life cycle, making user's data information more accurate, continuous, and valuable, thus ensuring the accuracy of system's health assessment and early warning of user's health condition, achieving the goals of reduc- ing the risk state, preventing the occurrence of diseases, and improving life. This will reduce the risk status, prevent diseases, improve the quality of life, save medical resources, and reduce the cost of medical treatment for patients [10]. At the same time, the development of personal health management system is of great importance in improving the efficiency and quality of medical services, changing the medical model, and realizing scientific cost control and actuarial management. There are tens of thousands of personal health management application platforms in China, but most of them provide a single health management model, only providing the management of one or several health data [11]. There are also many health management application platforms that mainly focus on medical consumption and cooperate with medical institutions to provide online registration for medical treatment, etc., without providing precise health management modules to users [12]. These single-mode or paid health management platforms fail to create a complete health management mechanism for users, and the vast majority of existing health management systems fail to enter rural households, staying only for users with higher living standards.

This project is based on a student-oriented approach to implement a health management system based on the complete process of health management. The system is designed to assist students in the complete process of detecting, evaluating, and guiding themselves on potential risk factors for their health. It is a professional system belonging to the medical field. The purpose of the system is to motivate students to implement autonomous health management, i.e., students improve their ability to manage their own health through multiple ways such as self-learning, self-building, and selfestablishing databases and rely on their own strength to monitor and care for their health indicators in real time, which is a dynamic autonomous health management model. From the student level, the use of health management system can reduce the expenditure of medical expenses; most students are provided with good health maintenance and protection services, then the risk factors of diseases can be controlled within a certain range without clinical reactions. The use of health management systems can reduce students' health risk factors, making it a virtuous circle in which students become more and more aware of their health management, and independent health management makes students secure.

\section{Related Work}

As early as 1929, world's first health management organization was established in Los Angeles, USA, which proposed a 
comprehensive approach to health management by focusing on prevention to reduce the incidence of disease and conducted practical exploration of health management. In the early 1970s, the U.S. government formulated rules and recommendations for health maintenance and established and passed a health maintenance bill for this purpose. In the following years, the bill was implemented by establishing health research centers and developing health assessment [13]. In the following years, the act was implemented by establishing health research centers, developing health assessment tools, and so on. In 2010, the U.S. government launched a Decade of Health program to improve national health and disease prevention awareness [14]. Japan's health care system features private, one-on-one, long-term health follow-up assessments [15]. This tracking and assessment service is family-based, with a comprehensive health record that is updated and maintained over time, and recommendations for assessment are made accordingly.

The business of health management has been very mature in foreign countries, and with the expansion of the scope of the industry and changes in the composition of the disease, many countries have invested in the management of chronic diseases and other modern health persistent disease research, through sensors, the Internet, and other technologies to establish a sound personalized chronic disease monitoring system [16]. The concept of health management emerged in the United States and other Western countries in the 1990s and was first introduced by the American insurance industry. Today, the United States is still the global leader in healthcare development, especially in the field of health management [17]. The closed-loop health management system mainly targets people with subhealth or subdisease, through the steps of health data collection, health assessment, plan making, guidance intervention, and user feedback, cultivating users' good living habits, improving their self-health management awareness and self-healing power, and achieving the effect of controlling the occurrence or deterioration of diseases at the root. In addition to the United States, health management is also developing rapidly in developed countries such as the United Kingdom, Japan, and Korea. With the development of Internet communication technology, many health management applications have emerged with electronic products such as computers and cell phones, mainly including platform applications, website applications, and cell phone applications. These health management applications with health data recording, online health consultation, and health condition assessment make health management no longer restricted by time and place and give greater play to the value of medical resources, achieving the effect of reducing disease rates and improving the quality of life [18]. As governments continue to launch exploratory national health management construction documents and plans, major Internet companies have responded to the development of the times and have invested in research in the health management field.

So far, many advanced and professional health management applications have been launched abroad. These include the platform applications Health Kit and Google Fit, the website application Health Vault, and a large number of cell phone applications, among which cell phone applications have the advantages of portability and easy operation and are most easily received and used by people [19].

Medisafe reminds users to take their medication on time through push messages and prompts them to record the amount of medication they take when they take it [20]. If the system does not detect that the patient has taken the medication, patient's friends and family are notified and can take action against the patient. In addition, Sweetch, an Israeli company, has developed a disease risk diagnosis and prevention software, which can predict user's chances of getting sick and make users who are already sick but do not know if they are aware of their condition. In addition to Western countries, health management systems are also growing rapidly in Asian countries such as Korea and Japan. Samsung's S Health has become one of the most accepted personal health management platforms in Korea today. $S$ Health mainly includes environmental detection, exercise management, and health diary, through which users can record their daily health activities and customize their health and exercise plans to accomplish their goals under the guidance of the system [21]. Physical health data such as heart rate can also be measured through the sensors built into the Samsung Galaxy and Gear. In 2017, Japanese healthcare company OmronHealthcare invested in Kardia Pro, a heart disease prevention management software from Alivecor Alive Cor, which tracks users' ECG, weight, blood pressure, daily behavior, etc., uses artificial intelligence technology for data analysis and comparison to identify potential risk factors that are not detected by doctors, and organizes the results in user's personal health profile to help doctors diagnose the user in case of sudden illness.

\section{Big Data-Based Physical Health Management Model}

3.1. A Closed-Loop Health Management Model Based on Big Data. Closed-loop management is a complete management system, first proposed by Robert S. Kaplan, founder of the Balance Card, and initially applied to improve the efficiency of company management and operations. With the development of health management field, the traditional simple health management model cannot meet the needs of people's health management, and closed-loop management in health management has been widely used. Closed-loop health management mainly refers to the process of decision-making, implementation, feedback, reimplementation, and feedback in health management, so that personal physical quality can be continuously improved in the cycle of accumulation and promote the improvement or maintenance of personal health level. The closed-loop health management system mainly targets people with subhealth or subdisease, through the steps of health data collection, health assessment, plan making, guidance intervention, and user feedback, cultivating users' good living habits, improving their self-health management awareness and self-healing power, and achieving the effect of controlling the occurrence or deterioration of diseases at the root. Professor Pan Xiaojue from the Shanghai Jiaotong University pointed out that 


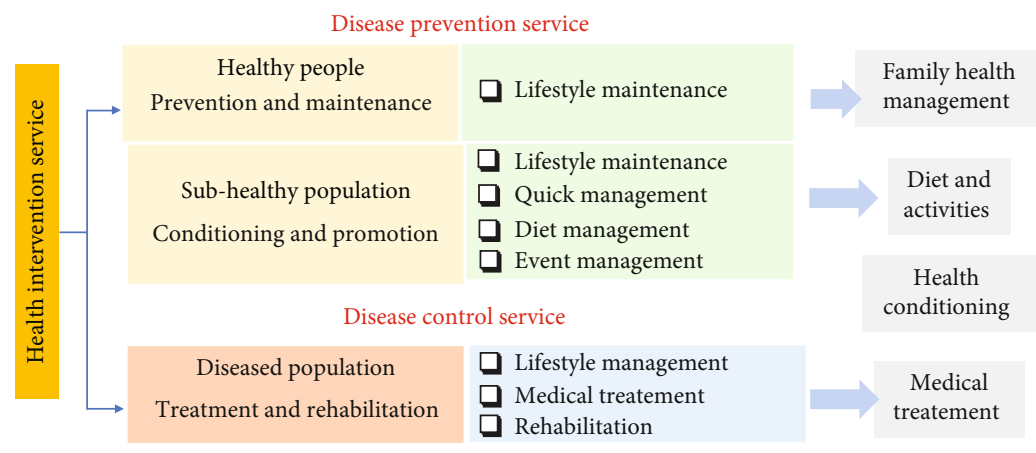

Figure 2: Closed-loop health management model.

health management mainly starts from three closed loops, the first one is the closed loop of testing means, body's physiological indicators are constantly changing, and in the process of health management, physiological indicators are constantly tested to find out which physiological indicators have problems, so as to solve problems. The second is the closed-loop intervention path; closed-loop health management system is mainly used by people with chronic diseases, not only from the consideration of improving the disease but also through the system to continuously intervene to guide the lifestyle and gradually improve the root cause of the disease. The third is the closed loop of repairing raw materials. The repairing raw materials of chronic diseases and subhealthy people are mainly their own health management consciousness and mentality, and a good health management consciousness is a strong immunity and self-healing power. Zhang Juan et al. from the Tongji Medical College conducted a study on closed-loop health management for diabetic people, proposing a dynamic closed-loop diabetes health management model of prehospital prevention-inhospital education-posthospital rehabilitation through online diagnosis and treatment, medication guidance, and access to or sharing of health data, etc.

The researchers explored the information-based closedloop health management model for subhealthy people under the Internet+ model, in which medical and nursing personnel realize long-term intelligent closed-loop health management for subhealthy people in and out of the hospital through health data monitoring [22], trend analysis, guidance intervention, and online tracking and management. The PDCA management cycle, proposed by Dr. Hart, an American quality management expert, divides management into four stages: plan, do, check, and act. The PDCA management cycle is a very effective management model for all kinds of management, which can develop people's own management awareness and make users develop the instinct of automatic management through the steps of planning guidance and result checking. The PDCA management cycle is applied to personal health management system, which is the basic management model of personal health management system based on multidimensional data model proposed in this paper, to achieve closed-loop personal health management through the cycle of data collection, data collation, health assessment, guidance intervention, and imple- mentation of programs. The personal health management model proposed in this paper is shown in Figure 2.

3.2. Big Data Collection. Standardized big data collection and recording methods are the basis for health management of chronic diseases and subhealthy people and are the basic requirements for data collation and analysis, health assessment, personalized health intervention, and management effect evaluation. There are various channels and types of health data collection, mainly from professional clinical data of medical institutions, health data integrated by regional health service platforms, conventional health data collected by portable wearable devices, and users' self-quantified health data. The clinical data of medical institutions refers to professional medical health data, which includes medical data generated from various medical clinical consultations and medical examinations, and these health data are the most valuable data in users' health management. Regional health service platform fully brings together personal health data from many medical institutions of users, which at this stage mainly refers to medical checkup data. Portable wearable devices mainly collect users' ECG, blood pressure, blood oxygen, sleep, pulse, body temperature, and other physical signs data. User self-quantified health data includes exercise information, diet, smoking, and drinking. Self-quantified data is also an important basis for systematic health assessment and disease judgment, which helps to provide personalized health guidance and intervention to users. These health data are generally divided into three formats: structured, semistructured, and unstructured. In this paper, by collecting the above channels and types of health data, we analyze and mine the health data through distributed data processing technology and perform trend analysis, health prediction, and risk warning on user's health status in the system.

Based on health data sources, this paper is designed to obtain users' health information through the following three paths: PC-based website system, mobile-based APP platform, and smart wearable devices.

\section{(1) PC-based website system}

According to the classification, format, and other data attributes of health data, we design various health data 
record forms based on the PC-based health management website. The collection method based on the PC-based website is based on user-initiated entry, and the basic principles of health data collection refer to the relevant regulations in the Basic Architecture and Data Standards for Health Records and Basic Architecture and Data Standards for Electronic Medical Records issued by the Ministry of Health of China. Among them, the basic personal information collection form refers to "HRA00.01 Basic Data Set Standard for Personal Information," the medical physical examination data entry form refers to "HRC00.04 Basic Data Set Standard for Adult Health Examination," the hypertension collection record module refers to "HRB04.01 Basic Data Set Standard for Hypertension Case Management," and the diabetes collection record module refers to "HRB04.02." The other health data collection record modules are also designed according to the data format and attributes in the standard.

\section{(2) Mobile-based APP platform}

The main form of the personal health management system described in this paper is based on the mobile APP platform, and this health management system has developed APPs based on both Android and iOS in order to facilitate users to record and manage their health at any time. In addition to the PC-based website system, the mobile APP is designed with interfaces for unstructured data voice, image, audio, and video collection. The database between the PCbased website system and the mobile-based APP is synchronized through the server database, which facilitates user records and unified data management.

\section{(3) Smart wearable-based devices}

Smart wearable devices are the main tools for daily monitoring of body data for patients with chronic diseases and are the main source of routine health data. Examples include real-time wearable health devices such as dynamic blood glucose monitors and ECG monitors, as well as nonrealtime monitoring devices such as pedometers and blood pressure monitors. The detected health information is transmitted and stored in the database of this system through the Bluetooth function that smart health devices have.

The design of the database model is the focus of this paper. The traditional personal health management system does not conduct in-depth research on the attributes of health data such as diseases and body organs. This paper proposes a personal health management system based on multidimensional data model, which adopts a snowflake multidimensional data model and mines the attributes of diseases, body organs, and clinical departments of health data according to relevant clinical diagnosis guidelines and health data standards issued by the Ministry of Health of China, so that users can manage health data, intelligent health assessment, and disease risk prediction from multiple dimensions such as diseases and body organs, fully reflecting the value of health. The value of health data is fully reflected.

The traditional personal health management system only collects a large amount of personal health data and makes a

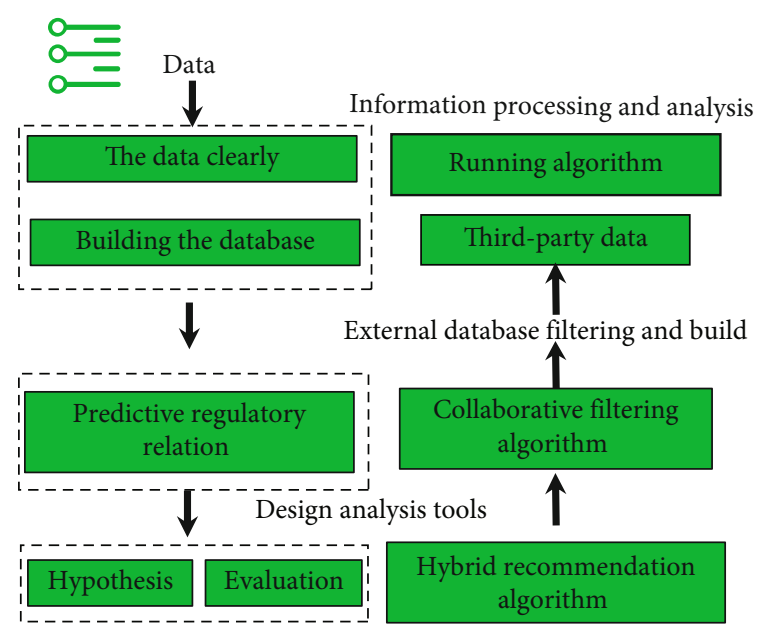

Figure 3: Multidimensional warehouse application for big data.

simple list, without taking into account the multiple attributes and dimensions of health data, so that the potential value of health data in health assessment and disease prediction cannot be fully utilized. Usually, a health data has multiple attributes and can be used for diagnosis of multiple body parts and disease types, for example, common blood pressure data; a large amount of blood pressure data can be used not only for judging the level of blood pressure but also for prediction assessment of cardiovascular system diseases combined with heart rate, daily symptoms and living habits, and for judging cerebrovascular health status combined with intraocular pressure and brain symptoms. In this paper, we design a multidimensional database model and set various attributes such as diseases and organs for each health data element, so that users can flexibly choose the use of health data and decide the role of data, so that health data can play a greater value in health assessment and disease prediction of personal health management system. In the personal health management system based on multidimensional data model, the relationship between each health data type needs to be standardized and designed according to the health assessment analogy, and clear and accurate data relationships and data attributes help to improve the decisionmaking accuracy of personal health management health assessment and disease prediction, etc. This paper designs a data warehouse with multidimensional data model, as shown in Figure 3, which mainly studies and solves the problem of obtaining information from the database. It features a topic-oriented, integrated, stable, and time-varying data collection, which is used to analyze data and discover new values of data, mainly for analytical reports and decision support in management, and meets the needs of user health data analysis and evaluation in health management systems.

\section{Artificial Intelligence-Based Health Fuzzy Integrated Assessment Model}

4.1. Health Assessment Index Weights. Indicator weights represent the importance of the indicator in health assessment, 


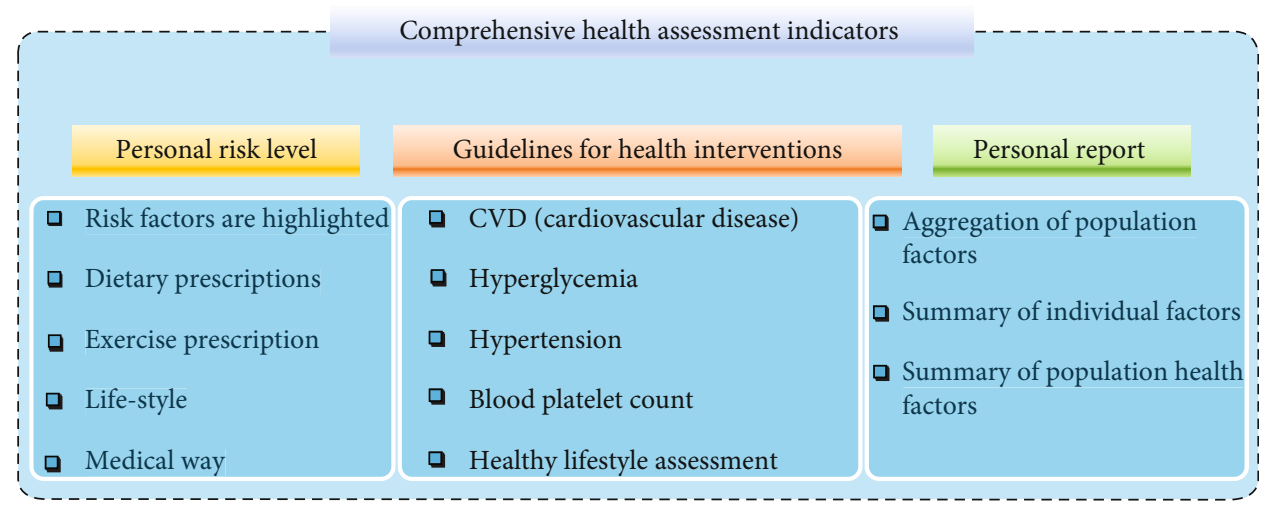

FIgURE 4: Hierarchy of health assessment indicators.

and reasonable allocation of each assessment indicator weight is the key to ensure the accuracy and scientificity of health assessment, and this paper selects hierarchical analysis method to obtain the assessment indicator weights. Hierarchical analysis was proposed by Thomas L. Saaty, an American operations researcher, in the 1970s. It is a combination of qualitative and quantitative decision analysis method, which can convert semiqualitative and semiquantitative problems into quantitative calculations. The health evaluation system built in this paper has both qualitative and quantitative evaluation factors, so using this method can hierarchize the complex medical health problem system and calculate the importance or weight value of each factor quantitatively by comparing the importance between related factors to establish a preliminary quantitative evaluation system. The main steps of the hierarchical analysis method to obtain the weights of evaluation indicators are as follows: firstly, the complex decision problem is stratified according to the general objective, criterion layer, and indicator layer, and then, the judgment matrix of each layer is determined, and then, the priority weights of each indicator in each layer to the elements of the upper layer are obtained by solving the eigenvectors of the judgment matrix and performing the consistency test.

\section{(1) Layering}

According to the hierarchical analysis method, the hierarchical structure of the comprehensive health assessment index system was determined. As shown in Figure 4, the hierarchical structure of health assessment indexes is divided into target layer A; criterion layer B, with two categories; primary index layer $\mathrm{C}$, with 9 items; and secondary index layer D, with 6 items.

(2) Constructing the judgment matrix

After completing the evaluation model of evaluation indicators, the indicators within each level are analyzed and compared with each other, and the judgment matrix of the corresponding level is constructed. Judgment matrix construction method: select a layer of indicators in the hier- archy, and experts compare the importance of the indicators in that layer relative to the indicators in the upper layer two by two and record the comparison results in the form of a matrix, which can constitute the judgment matrix between two levels. In this paper, 10 experts are selected to participate in the comparison of the importance of each index, taking into account the relevance of experts' research field to the topic, the time of experts' practice, and the age distribution of experts. In this paper, 1-9 scale is used to quantitatively scale the importance between two indicators, and 1, 3, 5, 7, and 9 are used to indicate the importance of one indicator over another, and the larger the number means the more important it is; when an indicator is less important than another indicator, the reciprocal of $1,3,5,7$, and 9 is used to indicate the less important degree; when the hierarchical degree needs higher precision, the real numbers between 1 and 9 can be interpolated to improve the precision.

The judgment number $a_{i j}$ represents the assignment of the importance of index $A_{i}$ over $A_{j}$, and the judgment matrix of each layer of order $n n^{*}$ is formed by $a_{i j} .10$ experts determine the judgment numbers of two comparisons of each factor between the indexes of a layer according to the scale of 19 and average the judgment numbers given by the experts to obtain the judgment matrix of each layer as shown in

$$
\begin{aligned}
& Q= {\left[\begin{array}{cccc}
q_{11} & q_{12} & \cdots & q_{1 i} \\
\cdots & & & \\
q_{i 1} & q_{i 2} & \cdots & q_{i i}
\end{array}\right], } \\
& Q_{|A-B|}=\left[\begin{array}{c}
22 \\
11 / 2
\end{array}\right], \\
& Q_{|c-B|}=\left[\begin{array}{cccc}
2 & 2 & 1 & 1 / 5 \\
1 & 1 / 2 & 3 & 1 / 4 \\
2 & 3 & 4 / 5 & 6 \\
1 / 3 & 3 & 2 & 1
\end{array}\right] .
\end{aligned}
$$


(3) Hierarchical single ordering and its consistency detection

After constructing the judgment matrix, the hierarchical single ranking and consistency verification are performed according to the judgment matrix, and the maximum eigenvalue $\lambda_{\max }$ of each layer judgment matrix is found out by MATLAB, and the normalized eigenvector of the matrix is found out, and each component $\lambda_{i}$ of the eigenvector is the important ranking weight of this layer index relative to the upper layer index. In order to reflect the rationality and objectivity of the indicator weights, this paper conducts consistency verification for each judgment matrix constructed to test whether the matrix of each layer meets the requirements. The steps of the consistency test are as follows:

$$
c_{I}=\frac{\left(\lambda_{\mathrm{MAX}}-n\right)}{(\sqrt{n+m})} .
$$

The deviation consistency index indicates the degree of deviation of the judgment matrix from the consistency matrix, where $\lambda_{\max }$ denotes the maximum eigenvalue of the judgment matrix and $n$ denotes the dimensionality of the judgment matrix:

$$
C r=\frac{c_{I}}{R}=\frac{\left(\lambda_{\mathrm{MAX}}-n\right)}{R(\sqrt{n+m})} .
$$

Since the variability among the layers increases with the superposition of the layers, it is also necessary to conduct a consistency test on the total ranking, and the weight values that pass the total ranking consistency test can be determined to be adopted. The total ranking consistency test method: let the matrix of the relevant evaluation indexes $M_{i}(i=1,2, \cdots, n)$ in $M+1$ layer and $M$ layer pass the test of the matrix passed the single-sort consistency test, the single-sort deviation consistency index of the matrix is $\mathrm{CI}_{i}$, the average random consistency index is $\mathrm{RI}_{i}$, and the weight value of $M_{i}$ is $i$. Then, the relative consistency index of the total sort in $M$ layer can be calculated by the formula:

$$
R=\sum_{i=1}^{n} \frac{\mathrm{Cr}_{i}}{\mathrm{Rw}_{i}}
$$

Bringing in the values to calculate the relative consistency index of the primary indicator layer relative to the criterion layer, the results are shown in

$$
R=\frac{0.1}{0.9}+\frac{0.2}{0.5}+\frac{0.3}{0.6}=1.02 .
$$

Fuzzy comprehensive evaluation refers to the overall evaluation of things that are affected by multiple evaluation factors and have fuzzy nature by using the idea of fuzzy mathematics. It has the characteristics of clear purpose, strong system, and clear structure and can reasonably quantify the fuzzy things that are difficult to evaluate objectively in real life. In this paper, we choose the fuzzy comprehensive

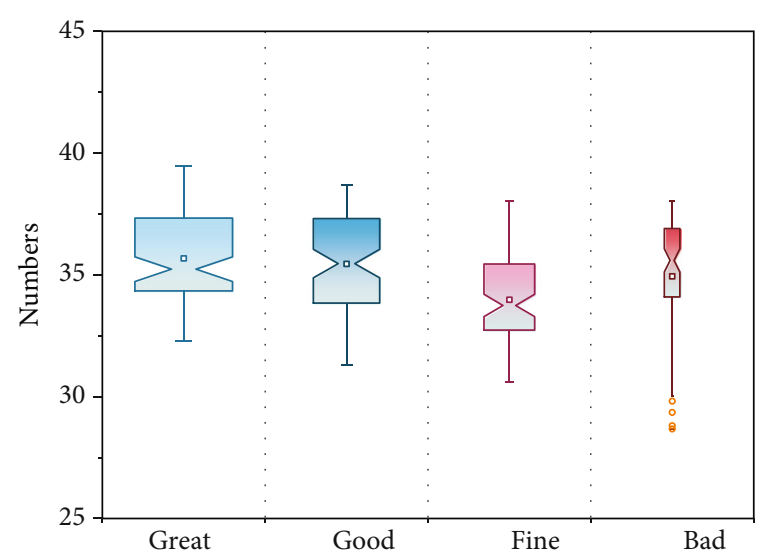

FIgURE 5: Diagram of blood glucose subordination and subordination function cut-off points.

evaluation method to evaluate the human health status. After obtaining the evaluation index weights by the above hierarchical analysis method, we use the affiliation function to construct the fuzzy matrix, the index weight set and the fuzzy evaluation matrix constitute the fuzzy subcomprehensive evaluation set, and finally, according to the principle of maximum affiliation, we get the health evaluation results. In this paper, we choose to construct the trapezoidal distribution affiliation function according to the data characteristics of health assessment indexes. The following is an example of blood glucose index to analyze the affiliation function, as Figure 5 shows the diagram of blood glucose affiliation and affiliation function dividing point.

The affiliation function with the rank of superior: here, the $Q$ means the judgment matrix, and the $q 1$ and $q 2$ means the judgment constant, which can be obtained from Figure 5:

$$
Q= \begin{cases}1, & x \leq q_{1}, \\ \frac{\left(q_{2}-q_{1}\right)}{2}, & q_{1}<x<q_{2}, \\ 0, & x \leq q_{2} .\end{cases}
$$

The affiliation function for a grade of good:

$$
Q= \begin{cases}2, & x \leq q_{1}^{\prime}, \\ \frac{\left(q_{2}^{\prime}-q_{1}^{\prime}\right)}{2}, & q_{1}^{\prime}<x<q_{2}^{\prime}, \\ 1, & x \leq q_{2}^{\prime} .\end{cases}
$$

The rank is the affiliation function in:

$$
Q= \begin{cases}3, & x \leq q_{1}^{\prime^{\prime}}, \\ \frac{\left(q_{2}^{\prime \prime}-q_{1}^{\prime^{\prime}}\right)}{2}, & q_{1}^{\prime^{\prime}}<x<q_{2}^{\prime^{\prime}}, \\ 2, & x \leq q_{2}^{\prime{ }_{2}} .\end{cases}
$$



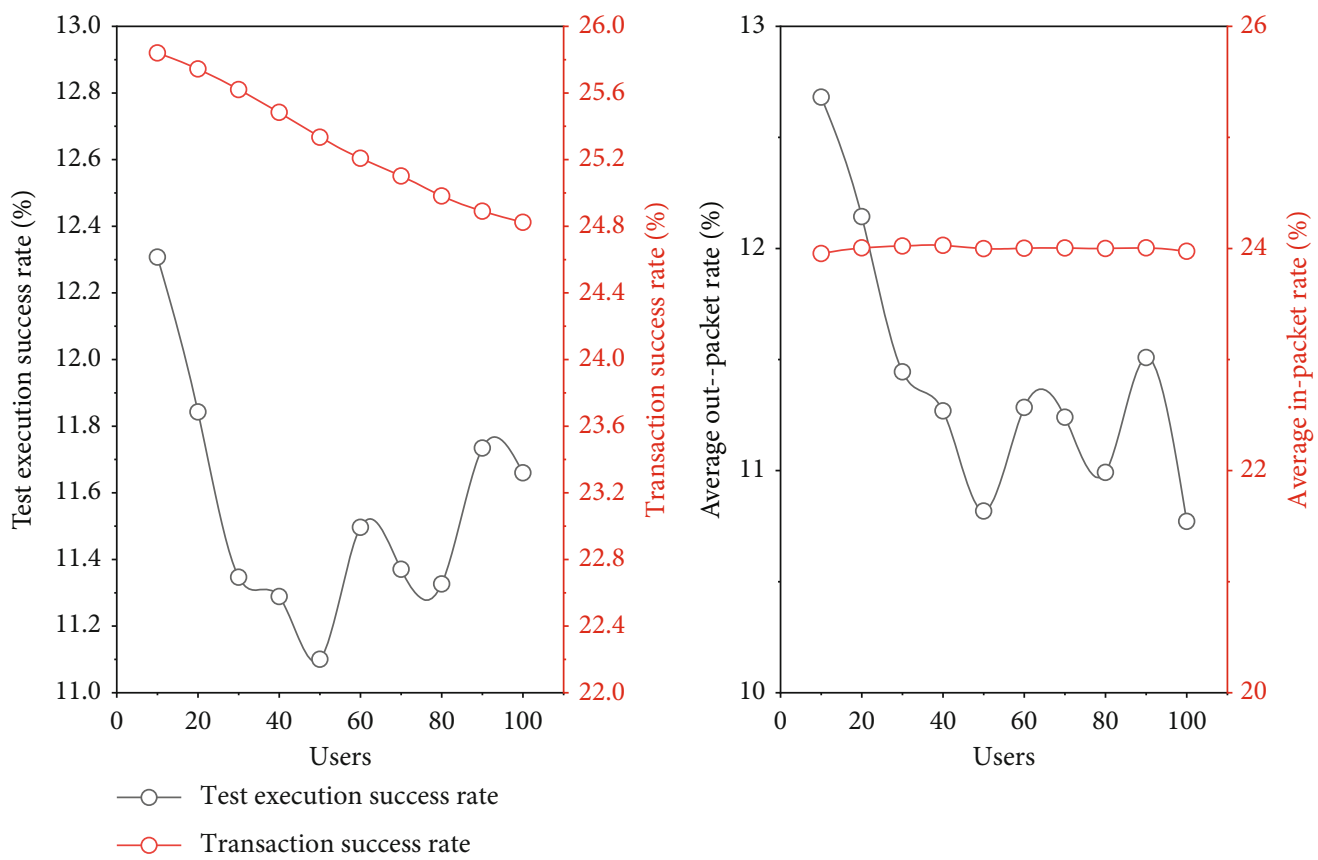

Figure 6: Performance test results under different load conditions.

The rank is a poor affiliation function:

$$
Q= \begin{cases}4, & x \leq q_{1}^{\prime \prime}, \\ \frac{\left(q_{2}^{\prime \prime \prime}-q_{1}^{\prime \prime \prime}\right)}{2}, & q_{1}^{\prime^{\prime \prime}}<x<q_{2}^{\prime \prime \prime}, \\ 3, & x \leq q_{2}^{\prime \prime \prime} .\end{cases}
$$

\section{System Testing}

5.1. System Performance Testing. The system was tested for software performance using Pressure Test Master, and the software performance such as the success rate of system time request, average transaction response time, packet sending rate, and packet receiving rate were tested, respectively. Meanwhile, the compatibility of the system was also tested, and the compatibility and adaptability of APP were tested using different brands of smartphones, respectively.

Figure 6 shows the summary of user system performance test results when the load concurrent users are 10, 20, 30, and 50, and the test results in the table show that the system transaction response success decreases slightly as the concurrent users increase, but the overall response time is above $90 \%$; the average transaction response time is less than $1 \mathrm{sec}-$ ond, which indicates that the system response speed is stable; the packet sending rate and packet receiving rate show a positive relationship, and the packet receiving rate continues to increase as the hit rate increases. As the hit rate increases, the packet receipt rate continues to increase, indicating that the server can respond to user event requests in a timely manner. The system responds well to the concurrent access of multiple users and meets the performance requirements of the user software. In this paper, the comprehensive health assessment model is studied and applied, and the fuzzy comprehensive assessment algorithm based on AHP is used to design the comprehensive health assessment model of this system, and the assessment model is tested using examples.

5.2. Health Data Collation. Health data recording is the C (check) step in the PDCA closed-loop health management model, and it is also a common function module for users to use the system on a daily basis. Users of the system can record health data through two channels: the website on the PC side and the APP health record on the mobile terminal. In addition to the text input on the website, the mobile terminal APP data recording method also has picture upload, Bluetooth import, voice recognition, and other recording methods. Figure 7 shows an example of the classification of the health data recording module in APP terminal, which is the same as the classification basis and database table in website terminal. According to user's demand for one-stop health management, a closed-loop health management model based on the PDCA cycle management model is adopted to design and implement the system functions from four aspects: health data collection and recording, health assessment, health plan, and tracking and execution.

The health plan is the P (plan) step in the PDCA closedloop health management model. The user makes a health improvement plan based on the health assessment results obtained in the above three ways. The plan form includes the contents that need to be recorded for a long time, such as the plan name, target data elements, and target values, and the contents that can be tracked and reminded by the system, such as the number of exercise steps and the name and time of medication. The system judges the 


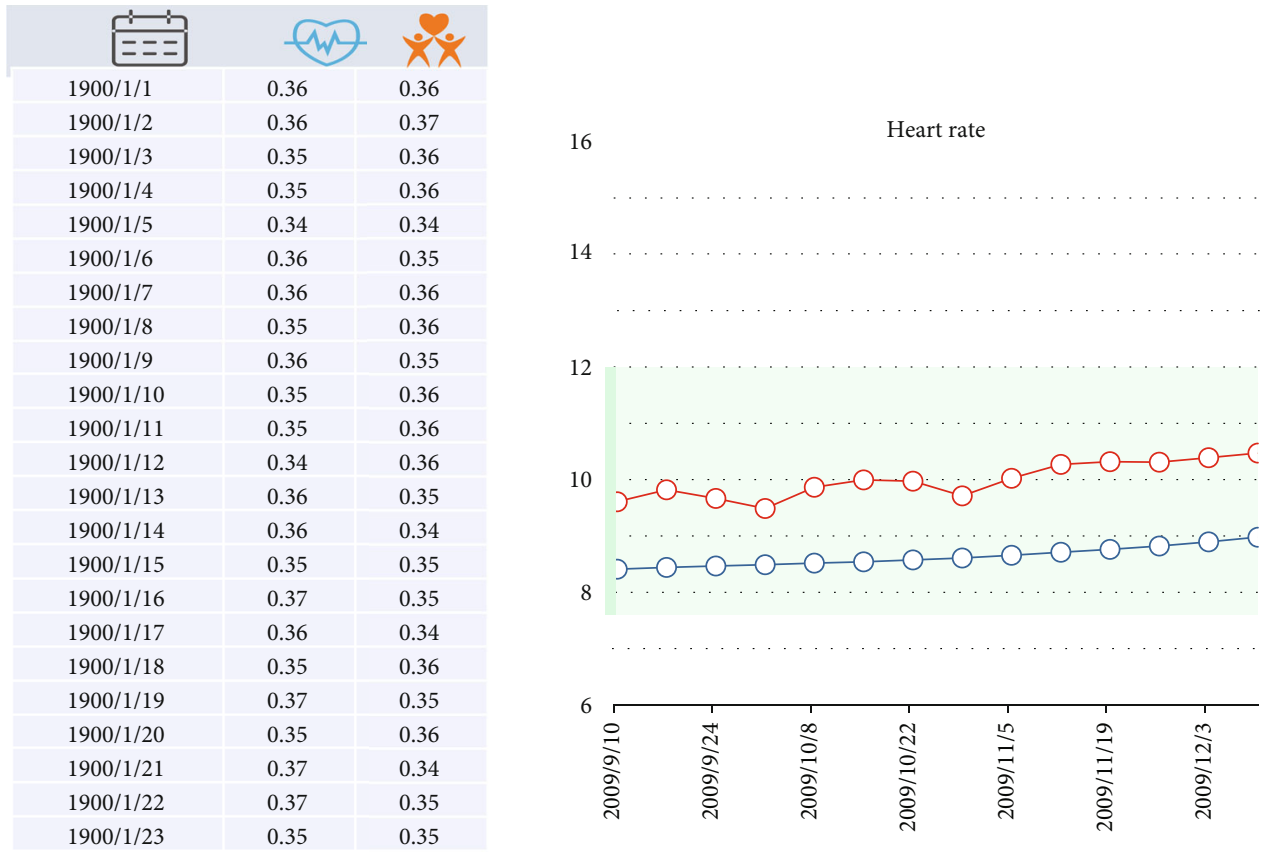

FIGURE 7: Health data summary presentation.

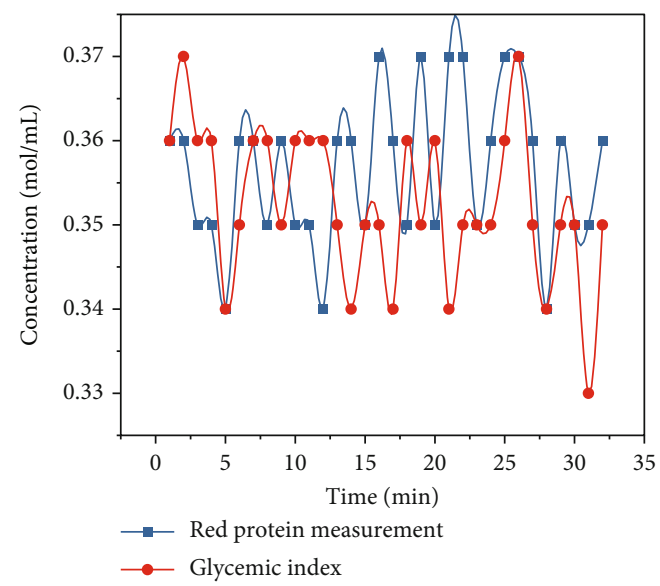

FIgURe 8: Glycemic index change graph.

reasonableness of user's health plan according to user's specific health condition and guides the user to make a suitable health plan. Figure 8 shows the effect of developing a diabetes control management plan. User's health plan is generated into a plan book, and the system will detect the items in the plan book that need to be tracked and reminded and then realize the next step of tracking and execution.

\section{Conclusion}

With the increasing incidence of chronic diseases in China, people's awareness of chronic disease management has been strengthened, and the emergence of personal health management systems is the general trend. At present, most of the health management systems in China have a single function and cannot meet the needs of long-term health management of users. In this paper, we summarize the shortcomings of existing health management systems and the needs of chronic disease population health management, and design and implement a personal health management system based on multidimensional data model. The system can meet users' various health management needs, create personalized health management guidance program for users, and achieve the purpose of improving users' health in all aspects. The average transaction response time is less than 1 second, which indicates that the system response speed is stable; the packet sending rate and packet receiving rate show a positive relationship, and the packet receiving rate continues to increase as the hit rate increases. According to user's demand for one-stop health management, a closed-loop health management model based on the PDCA cycle management model is adopted to design and implement the system functions from four aspects: health data collection and recording, health assessment, health plan, and tracking and execution. In this paper, the comprehensive health assessment model is studied and applied, and the fuzzy comprehensive assessment algorithm based on AHP is used to design the comprehensive health assessment model of this system, and the assessment model is tested using examples. This paper uses software development and database development techniques to design and implement this system, initially realizing the functions of login and registration, health data collection, health assessment, health planning, and tracking execution. The functions and performance of the software system were also tested, and the test results showed that this system basically meets user's needs for a health management system.

In the future, we would explore the information-based closed-loop health management model for subhealthy people under the Internet+ model, in which medical and nursing personnel realize long-term intelligent closed-loop 
health management for subhealthy people in and out of hospital through health data monitoring, trend analysis, guidance intervention, and online tracking and management.

\section{Data Availability}

The data used to support the findings of this study are included within the article.

\section{Conflicts of Interest}

The author declares that there is no conflict of interest.

\section{References}

[1] J. Wang, Z. Xie, Y. Li et al., "Relationship between health status and physical fitness of college students from south China: an empirical study by data mining approach," IEEE Access, vol. 8, pp. 67466-67473, 2020.

[2] F. Zhao, S. Sun, J. Xiong, and G. Zheng, "The effect of Baduanjin exercise on health-related physical fitness of college students: study protocol for a randomized controlled trial," Trials, vol. 20, no. 1, pp. 569-569, 2019.

[3] X. Lu, C. Yang, Y. Zhang et al., "Test method for health-related physical fitness of college students in mobile internet environment," Mathematical Biosciences and Engineering, vol. 16, no. 4, pp. 2189-2201, 2019.

[4] S. Mal-Ryun, "The comparative analysis of skeletal muscle mass, basal metabolic rate abdominal obesity and health related physical fitness of female college students - targeting students who majored in dance and general," Official Journal of the Koeran Society of Dance Science, vol. 37, no. 1, pp. 2938, 2020.

[5] T. Singh, "Impact of yogic asanas on the health-related physical fitness of students of agricultural biotechnology college," International Journal of Physical Education, Sports and Health, vol. 4, no. 1, pp. 2250-2253, 2019.

[6] C. B. Yang and T. H. Tsao, "Cardiorespiratory fitness, healthrelated physical fitness and academic performance in college students," Advances in Physical Education, vol. 10, no. 1, pp. 42-53, 2020.

[7] M. Sawant, "Efficacy of yogic practices for the promotion of academic achievement, mental health and health related physical fitness of college level female students," International Journal of Yoga, Physiotherapy and Physical Education, vol. 4, no. 4, pp. 14-17, 2019.

[8] T. S. Eshete, W. Mekonen, and H. Derseh, "Comparative assessment of vegetarian and non-vegetarian diets with physical fitness on body composition and lipid profiles among students at School of Medicine, College of Health Sciences, Addis Ababa University," Asian Journal of Medicine and Health, vol. 13, no. 4, pp. 1-15, 2019.

[9] P. Tang, Y. Wang, and N. Shen, "Prediction of college students' physical fitness based on K-means clustering and SVR," Computer Systems: Science \& Engineering, vol. 35, no. 4, pp. 237246, 2020.

[10] P. Bo, "Analysis and intervention on the influencing factors of college students' physical fitness," Revista Brasileira De Medicina Do Esporte, vol. 27, pp. 11-13, 2021.

[11] J. L. Wang, "The association between physical fitness and physical activity among Chinese college students," Journal of American College Health, vol. 67, no. 6, pp. 602-609, 2019.
[12] T. Pan, "An improved a priori algorithm for association mining between physical fitness indices of college students," International Journal of Emerging Technologies in Learning (IJET), vol. 16, no. 9, pp. 235-246, 2021.

[13] C. Qian, "Changes in body composition, blood pressure, bone density and physical fitness of female college students by wearing wearable devices for 24 weeks," The Korean Society of Sports Science, vol. 29, no. 1, pp. 683-694, 2020.

[14] S. I. Mushtaq and A. Kumar, "Comparative study of selected physical fitness components between urban and rural college level students," International Journal of Physical Education, Sports and Health, vol. 4, no. 1, pp. 2403-2406, 2019.

[15] T. M. Barnett, A. McFarland, J. W. Miller, V. Lowe, and S. S. Hatcher, "Physical and mental health experiences among African American college students," Social Work in Public Health, vol. 34, no. 2, pp. 145-157, 2019.

[16] D. A. Kinney, L. A. Nabors, A. L. Merianos, and R. A. Vidourek, "College students' use and perceptions of wearable fitness trackers," American Journal of Health Education, vol. 50, no. 5, pp. 298-307, 2019.

[17] J. Mora-Gonzalez, I. J. Pérez-López, and M. Delgado-Fernández, “The '\$in TIME' gamification project: using a mobile app to improve cardiorespiratory fitness levels of college students," Games for Health Journal, vol. 9, no. 1, pp. 37-44, 2020.

[18] M. Wang, Y. Guo, Y. Zhang et al., "Promoting healthy lifestyle in Chinese college students: evaluation of a social media-based intervention applying the RE-AIM framework," European Journal of Clinical Nutrition, vol. 75, no. 2, pp. 335-344, 2021.

[19] P. Prieto-González and J. Sedlacek, "Physical education: practice, cessation and resumption in untrained Saudi college students," in Series Physical Education and Sport, pp. 681-692, Facta Universitatis, 2021.

[20] N. A. Mir, "Comparative study of health related physical fitness of college level students of volleyball and football players," International Journal of Physical Education, Sports and Health, vol. 7, no. 6, pp. 222-224, 2020.

[21] J. DiMatteo, C. Radnitz, K. L. Loeb, and J. Ni, “The application of optimal defaults to physical education courses in college students: a simulation study," Journal of Teaching in Physical Education, vol. 38, no. 4, pp. 393-397, 2019.

[22] L. Yu, S. Tao, W. Gao, and L. Yu, "Self-monitoring method for improving health-related quality of life: data acquisition, monitoring, and analysis of vital signs and diet," ASP Transactions on Pattern Recognition and Intelligent Systems, vol. 1, no. 1, pp. 24-31, 2021. 